\title{
Contaminación por mercurio de leche materna de madres lactantes de municipios de Antioquia con explotación minera de oro
}

\author{
Carlos Federico Molina ${ }^{1,2}$, Catalina María Arango ${ }^{3}$, Hernán Sepúlveda1 \\ 1 Grupo en Salud y Seguridad en el Trabajo, Facultad Nacional de Salud Pública, Universidad de Antioquia, \\ Medellín, Colombia \\ 2 Grupo de Investigación BISMA, Tecnológico de Antioquia, Medellín, Colombia \\ 3 Grupo de Epidemiología, Facultad Nacional de Salud Pública, Universidad de Antioquia, Medellín, Colombia
}

Introducción. La leche materna es esencial para el desarrollo del ser humano, pero puede contener sustancias tóxicas provenientes de la contaminación ambiental, especialmente en las áreas mineras. Objetivo. Determinar la prevalencia de la contaminación con mercurio de la leche materna de mujeres lactantes residentes en los municipios con explotación minera de oro.

Materiales y métodos. Se hizo un estudio transversal de 150 madres lactantes de cuatro municipios mineros de Antioquia (El Bagre, Segovia, Remedios y Zaragoza), a quienes se les hizo una encuesta sobre factores sociodemográficos, ocupacionales y ambientales relacionados con el mercurio, y se les tomaron muestras de leche materna, de orina y de cabello. Se calculó el promedio de la concentración de mercurio y las prevalencias municipales de contaminación.

Resultados. El promedio de la concentración de mercurio en la leche materna fue de 2,5 ( \pm desviación estándar 9,2) $\mu \mathrm{g} / \mathrm{L}$. La prevalencia de muestras de leche materna con niveles altos de mercurio fue de $11,7 \%$.

Conclusión. En este estudio se evidencia un grave problema en las regiones mineras auríferas de Antioquia por el efecto de la contaminación con mercurio en sectores de la población más vulnerable.

Palabras clave: mercurio; contaminación; leche humana; lactancia materna; minería; oro; Colombia. doi: https://doi.org/10.7705/biomedica.v38i0.3609

Mercury contamination in breast milk of nursing mothers in gold mining municipalities of Antioquia, Colombia

Introduction: Breast milk is essential for human development, but it may contain toxics derived from environmental pollution, especially in mining areas.

Objective: To assess the prevalence of mercury contamination in breast milk and factors associated with its transfer to nursing mothers living in municipalities with gold mining.

Materials and methods: We conducted a cross-sectional study with 150 nursing mothers in four municipalities of Antioquia (El Bagre, Segovia, Remedios and Zaragoza) with a mining tradition. We surveyed these mothers to obtain information on their sociodemographic, occupational and environmental factors related to mercury, and we took breast milk, urine and hair samples. We calculated mercury level averages and the contamination prevalence per municipality.

Results: Mercury average in breast milk was 2.5 ( \pm standard deviation: 9.2$) \mathrm{mg} / \mathrm{L}$. The prevalence of mercury contamination in samples with a high level of mercury was $11.7 \%$.

Conclusion: This study shows that there is a serious problem of mercury contamination in gold mining regions of Antioquia, with significant involvement of the most vulnerable population.

Key words: Mercury; contamination; milk, human; breast feeding; mining; gold; Colombia. doi: https://doi.org/10.7705/biomedica.v38i0.3609

\section{Correspondencia:}

Carlos Federico Molina, Calle $62 \mathrm{~N}^{\circ} 52-59$, Medellín, Colombia Teléfono: (544) 219 6432; fax: (544) 2196800

carlosfedericom@gmail.com

Recibido: 15/09/16; aceptado: 15/03/17
La exposición a mercurio tiene efectos neurológicos a corto y a largo plazo, especialmente durante el periodo de la vida en la que los sistemas se encuentran en formación y desarrollo (1-6). En diversos estudios se ha detectado mercurio y otros

\section{Contribución de los autores:}

Todos los autores contribuyeron a la concepción y diseño del manuscrito; al análisis e interpretación de los datos; a la redacción del manuscrito y a la revisión crítica del contenido intelectual. 
metales pesados en mujeres gestantes y en la leche materna, la cual se ha determinado como la principal fuente de exposición a mercurio durante esta fase crítica del ciclo vital humano (6).

En las zonas de explotación minera de oro, la exposición al mercurio se deriva del uso de este tóxico durante el proceso productivo y extractivo del metal, ya que el material extraído de la mina se amalgama con mercurio para separar el oro del material menos valioso; esta amalgama se somete después a calcinación para liberar el oro, pero, también, produce vapores de mercurio que son inhalados por los mineros (7-11), los compradores de oro y la comunidad en general, incluidas las mujeres. Estos vapores de mercurio metálico son transportados a la atmósfera para después precipitarse y depositarse en los cuerpos de agua $(11,12)$, donde, por acción de los microorganismos, pasan por un proceso de metilación que los transforma en metilmercurio, el cual contamina los alimentos de consumo humano, especialmente el pescado $(7,8,13)$.

La utilización de mercurio en la explotación de oro en los municipios de Segovia, Remedios, Zaragoza, El Bagre y Nechí, ha convertido al departamento de Antioquia en el lugar con la mayor contaminación de mercurio per cápita en el mundo (14). En estos municipios se ha estimado que hay entre $15.000 \mathrm{y}$ 30.000 mineros artesanales de oro, además de los que trabajan con grandes compañías mineras, y que la producción fluctúa entre 10 y 20 toneladas de oro al año (2010), lo cual exige el uso de alrededor de 93 toneladas de mercurio (14). Se calcula, asimismo, que el $63 \%$ de la producción total de oro de Colombia se deriva de la explotación que se lleva a cabo en el departamento de Antioquia (15). En las ciudades, los niveles de mercurio en el aire están entre $300 \mathrm{ng} \mathrm{Hg} / \mathrm{m}^{3}$ y $1^{\prime} 000.000 \mathrm{ng} \mathrm{Hg} /$ $\mathrm{m}^{3}$ (mediciones hechas en los sitios de compra de oro), con un promedio de $10.000 \mathrm{ng} \mathrm{Hg} / \mathrm{m}^{3}$ en las áreas residenciales (14), niveles peligrosamente altos si se comparan con los $1.000 \mathrm{ng} \mathrm{Hg} / \mathrm{m}^{3}$ permisibles según la Organización Mundial de la Salud (OMS) $(14,16,17)$.

La leche materna es esencial para el desarrollo físico, mental y cognitivo del recién nacido, y previene un número significativo de enfermedades $(18,19)$. La OMS recomienda la lactancia materna exclusiva durante los primeros seis meses de vida $(20,21)$. Paradójicamente, a pesar de sus innegables beneficios, la leche materna puede contener sustancias tóxicas, como el mercurio, provenientes de la contaminación ambiental a la que se exponen las madres durante la gestación y en el periodo de lactancia, tanto, que se ha establecido como la principal fuente de exposición a metales pesados durante el periodo neonatal $(6,21)$. A pesar de la magnitud del problema de contaminación del aire por mercurio metálico en áreas de explotación de oro, en pocos estudios se ha valorado la presencia de esta sustancia tóxica en la leche materna en dichas zonas $(22,23)$. La mayoría de los estudios en que se ha evaluado el papel del mercurio durante las fases de gestación y lactancia, no se han hecho en comunidades dedicadas a la explotación minera sino en comunidades de pescadores en las que la principal fuente de exposición al mercurio son los peces y los alimentos contaminados con metilmercurio (20-24).

En este contexto, el presente estudio buscó determinar las concentraciones de mercurio total en la leche materna, la orina y el cabello de mujeres en periodo de lactancia residentes en municipios con explotación minera de oro en el departamento de Antioquia.

\section{Materiales y métodos}

\section{Diseño del estudio}

Se hizo un estudio de corte transversal en los municipios de Segovia, Remedios, Zaragoza y El Bagre, ubicados en zonas de explotación minera de oro a gran y pequeña escala en el departamento de Antioquia (Colombia). Segovia y Remedios están en el noroeste de Antioquia, y su principal actividad económica es la minería de oro, especialmente la minería de veta; en tanto que los municipios de El Bagre y Zaragoza están en el norte del departamento, a orillas del río Nechí, y su principal actividad económica es la minería de oro, especialmente la minería de aluvión (figura 1).

La población objeto de estudio estuvo constituida por mujeres en periodo de lactancia que llevaban residiendo en el área urbana de estos municipios durante, por lo menos, 12 meses y aceptaron participar en el estudio.

\section{Muestra}

Para la estimación del tamaño de la muestra, se consideraron los siguientes criterios: un nivel de confianza del $95 \%$, una proporción esperada de mujeres lactantes con concentraciones de mercurio por encima de lo permitido del $7 \%$ y una precisión del $4 \%$. Los datos para el cálculo del tamaño de la muestra se basaron en un estudio realizado en 


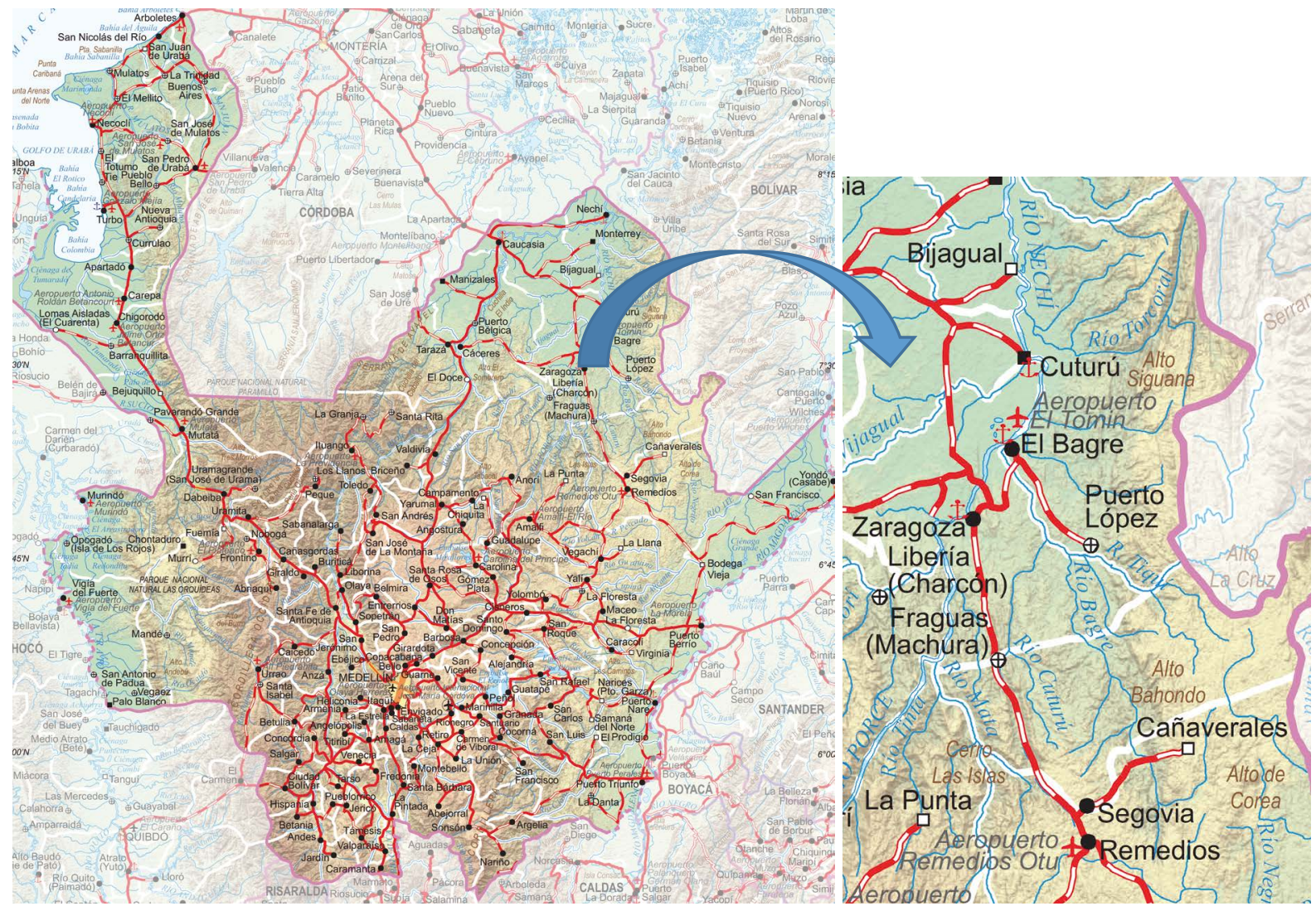

Figura 1. Mapa hidrográfico de la zona de estudio. Fuente: Instituto Geográfico Agustín Codazzi

tres áreas mineras de tres países diferentes (23). De este modo, el tamaño calculado de la muestra con el programa Epidat ${ }^{\mathrm{TM}}$, versión 4.0, fue de 150 mujeres.

El número de madres se asignó proporcionalmente por municipio, es decir, el porcentaje de participación de cada uno en el tamaño de la muestra fue igual a su participación en el tamaño de la población; así, se seleccionaron 55 madres en El Bagre, 50 en Segovia, 28 en Zaragoza y 17 en Remedios. Las 150 participantes se seleccionaron de manera directa con base en un censo en los hospitales de los cuatro municipios.

\section{Recolección de la información}

La recolección de los datos en campo se hizo mediante una encuesta de 80 preguntas sobre factores de riesgo de exposición relacionados con la ocupación o trabajo de las participantes y sobre factores ambientales. La encuesta sociodemográfica se ajustó a los criterios de encuestas similares utilizadas en los programas de evaluación del riesgo por exposición a mercurio de algunos programas de las Naciones Unidas $(24,25)$.
El investigador principal, con formación en salud ocupacional y toxicología, recolectó la información entre los meses de enero y mayo de 2015.

\section{Muestras de leche materna, orina y cabello}

Para medir la concentración de mercurio en la leche materna, se tomó una muestra de leche en el transcurso de los tres primeros meses a partir del parto: se le pidió a la madre lactante que recolectara $15 \mathrm{ml}$ de leche materna por escurrimiento a cualquier hora del día, en tubos de ensayo previamente tratados con ácido nítrico, procedimiento este utilizado en diversos estudios internacionales (26-32). Asimismo, las participantes tomaron las muestras de su orina según las instrucciones que se les impartieron, es decir, se recogió la primera orina de la mañana, en tanto que las muestras de cabello se tomaron en la región occipital siguiendo los procedimientos estándar de recolección de este tipo de muestras (33): se cortaron con tijeras de acero inoxidable entre $100 \mathrm{y}$ $200 \mathrm{mg}$ de cabello muy cerca del cuero cabelludo, las cuales se almacenaron en bolsas de polietileno individuales de cierre hermético de $5 \times 10 \mathrm{~cm}$. 
Una vez recolectadas las muestras, las bolsas se rotularon y se almacenaron en las neveras de los centros hospitalarios de cada uno de los municipios, para luego ser trasladadas en neveras portátiles a una temperatura de $4^{\circ} \mathrm{C}$ al Laboratorio de Toxicología y Gestión Ambiental de la Universidad de Córdoba en las siguientes 72 horas.

Las muestras de leche materna y de orina para el análisis de mercurio total, se homogeneizaron y se secaron, y las de cabello se lavaron y homogeneizaron. Posteriormente, las muestras se calcinaron a $650{ }^{\circ} \mathrm{C}$ en un horno de grafito; el mercurio liberado fue atrapado en un amalgamador de oro para, posteriormente, medirlo por espectrometría de absorción atómica, según el método EPA 7473 en un analizador directo de mercurio DMA 80 (34). El límite de detección de mercurio total en la leche materna y en la orina fue de $0,5 \mu \mathrm{g} / \mathrm{L}$ y, en el cabello, de $0,1 \mu \mathrm{g} / \mathrm{kg}$.

Para garantizar la confiabilidad de los resultados obtenidos en las distintas matrices, se establecieron diferentes criterios de control de calidad: una curva de calibración con un coeficiente mínimo de correlación de 0,999, el análisis de los blancos por cada lote de muestras, la verificación de los estándares de control, las pruebas por duplicado para evaluar la ausencia de vidrio en $10 \%$ de las muestras, así como pruebas en el Laboratorio de Aguas de Cartagena (Acuacar) y en el Laboratorio de la Universidad de Cartagena, los cuales cuentan con la acreditación ISO 17025:2005 y son reconocidos en análisis toxicológico.

Los límites permisibles para las diferentes muestras fueron de $4 \mu \mathrm{g} / \mathrm{L}$ para la leche materna (35), de $1 \mu \mathrm{g} / \mathrm{kg}$ para el cabello (36) y de $20 \mu \mathrm{g} / \mathrm{L}$ para la orina (37).

\section{Análisis de los datos}

La concentración de mercurio total en leche materna y los datos sobre las características personales, ocupacionales y ambientales, se expresaron como distribuciones de frecuencias y porcentajes. Asimismo, se calcularon la media, la mediana y la desviación estándar, y se verificó el supuesto de distribución normal de las variables mediante la prueba de Kolmogorov-Smirnov con corrección de Lilliefors.

La correlación entre la concentración de mercurio total en la leche materna y la registrada en la orina y el cabello, se calculó en correlaciones bivariadas mediante la estimación de los coeficientes de correlación de Pearson y Spearman.

\section{Consideraciones éticas}

El estudio fue aprobado por el Comité de Ética de la Facultad Nacional de Salud Pública de la Universidad de Antioquia. Las participantes aceptaron ser parte del estudio mediante la firma del consentimiento informado.

\section{Resultados}

Se evaluaron 150 mujeres lactantes, residentes en los municipios de El Bagre, Segovia, Zaragoza y Remedios; $50 \%$ de ellas había residido en el municipio durante 17 años o menos, 55,3 \% vivía en zonas de minería de aluvión y, 44,7\%, en zonas de minería de socavón. El $50 \%$ de las mujeres tenía 22 años de edad o menos, y la mayoría solo había estudiado hasta la secundaria; $78 \%$ de ellas estaban casadas o vivían en unión libre (cuadro 1).

Con respecto a las características de salud de las participantes, se observó que 56,7 \% eran multíparas y $18,7 \%$ tenían antecedentes de abortos. Cabe señalar que $38 \%$ de ellas había sufrido un episodio de malaria en el pasado y en el $44 \%$ se registró una mediana de tres amalgamas dentales.

En relación con los hábitos de salud, ninguna madre refirió fumar cigarrillos en el momento de la encuesta; $22 \%$ de ellas consumía licor y $27 \%$ hacía ejercicio regularmente, con una mediana de tiempo dedicado al ejercicio de tres días por semana (cuadro 1).

Al indagar por las condiciones ambientales y habitacionales en las que vivían las mujeres lactantes, se encontró que el material predominante de construcción de las viviendas era el ladrillo en las paredes, y la baldosa y el cemento en los pisos. La mayoría de las viviendas (68 \%) contaba con todos los servicios domiciliarios básicos: energía eléctrica, acueducto y alcantarillado; sin embargo, $18,7 \%$ de las mujeres manifestó que su vivienda no disponía de acueducto ni alcantarillado, 11,3\% de las viviendas solo contaba con energía eléctrica y tan solo $2 \%$ carecía de todos los servicios básicos.

En cuanto a la cercanía de la vivienda a los lugares de compra y de beneficio del oro, se evidenció que $15,3 \%$ de las mujeres vivía a menos de 50 metros de un lugar de compra de oro, $24 \%$, a dicha distancia de un lugar de beneficio del oro, y la mayoría de ellas pasaba todo el día en su hogar (cuadro 1).

En relación con la exposición ocupacional al mercurio en las madres lactantes, se determinó que $18,7 \%$ de ellas había trabajado con mercurio 
Cuadro 1. Características sociodemográficas, ocupacionales y ambientales de mujeres lactantes de los municipios de estudio

\begin{tabular}{|c|c|}
\hline Variable & n (\%) \\
\hline \multicolumn{2}{|l|}{ Municipio de residencia } \\
\hline El Bagre & $55(36,7)$ \\
\hline Zaragoza & $28(18,7)$ \\
\hline Segovia & $49(32,7)$ \\
\hline Remedios & $18(12)$ \\
\hline \multicolumn{2}{|l|}{ Estado civil } \\
\hline Casada/unión libre & $117(78)$ \\
\hline Soltera/separada & $33(22)$ \\
\hline \multicolumn{2}{|l|}{ Escolaridad } \\
\hline Estudios primarios & $34(22,7)$ \\
\hline Estudios secundarios & $105(70)$ \\
\hline Estudios tecnológicos y universitarios & $11(7,3)$ \\
\hline \multicolumn{2}{|l|}{ Consume licor } \\
\hline Sí & $33(22)$ \\
\hline No & $117(78)$ \\
\hline \multicolumn{2}{|l|}{ Hace ejercicio } \\
\hline Sí & $27(18)$ \\
\hline No & $123(82)$ \\
\hline \multicolumn{2}{|l|}{ Presencia de amalgamas dentales } \\
\hline Sí & $66(44)$ \\
\hline No & $84(56)$ \\
\hline \multicolumn{2}{|l|}{ Número de embarazos } \\
\hline Primípara & $65(43,3)$ \\
\hline Multípara & $85(56,7)$ \\
\hline \multicolumn{2}{|l|}{ Antecedentes de aborto } \\
\hline Sí & $28(18,7)$ \\
\hline No & $122(81,3)$ \\
\hline \multicolumn{2}{|l|}{ Antecedentes de malaria } \\
\hline Sí & $57(38)$ \\
\hline No & $93(62)$ \\
\hline \multicolumn{2}{|c|}{ Distancia entre la casa y el lugar de compra de oro más cercano (metros) } \\
\hline A menos o igual a 50 & $23(15,3)$ \\
\hline Más de 50 & $127(84,7)$ \\
\hline \multicolumn{2}{|c|}{ Distancia entre la casa y beneficidero del oro más cercano (metros) } \\
\hline A menos o igual a 50 & $36(24)$ \\
\hline Más de 50 & $114(76)$ \\
\hline \multicolumn{2}{|l|}{ Trabaja fuera del hogar } \\
\hline Sí & $17(11,3)$ \\
\hline No & $133(88,7)$ \\
\hline \multicolumn{2}{|l|}{ Antecedentes de trabajo con mercurio } \\
\hline Sí & $28(18,7)$ \\
\hline No & $122(81,3)$ \\
\hline \multicolumn{2}{|l|}{ Oficio del compañero o jefe de hogar } \\
\hline Minero & $91(60,7)$ \\
\hline No minero & $59(39,3)$ \\
\hline \multicolumn{2}{|l|}{ Quema mercurio en la casa } \\
\hline Sí & $39(26)$ \\
\hline No & $111(74)$ \\
\hline \multicolumn{2}{|l|}{ Almacena mercurio en la casa } \\
\hline Sí & $28(18,7)$ \\
\hline No & $122(81,3)$ \\
\hline
\end{tabular}

en el pasado, pero ninguna lo manipulaba en el momento de la encuesta; la mayoría de ellas había suspendido hacía un año los oficios relacionados con la manipulación de este metal. Además, se evidenció que el oficio más común en los compañeros permanentes o jefes del hogar era el de minero $(60,7 \%)$ y que $8 \%$ de las mujeres los ayudaban en sus labores. El $46 \%$ de las mujeres lavaba la ropa de toda la familia y solo $11,3 \%$ trabajaba fuera del hogar ocho horas diarias durante los siete días de la semana. Es importante resaltar que en $26 \%$ de los hogares de las mujeres se hacía quema de mercurio dentro de la vivienda y $18,7 \%$ lo almacenaba en sus casas (cuadro 1). 


\section{Mercurio total en la leche materna}

La concentración promedio de mercurio total en la leche materna fue de 2,11 $\mu \mathrm{g} / \mathrm{dl}( \pm 7,17)$. En $11,3 \%$ de las muestras de leche materna se encontraron niveles por encima de los permisibles (35). La concentración promedio de mercurio total en la leche materna en los municipios de El Bagre, Zaragoza, Segovia y Remedios, fue de $3,42 \mu \mathrm{g} / \mathrm{dl}( \pm 11,34)$, $0,8 \mu \mathrm{g} / \mathrm{dl}( \pm 0,63), 1,76 \mu \mathrm{g} / \mathrm{dl}( \pm 3,35)$ y $1,12 \mu \mathrm{g} / \mathrm{dl}$ $( \pm 0,71)$, respectivamente. En el municipio de El Bagre, $16,4 \%$ de las muestras de leche materna superaba los niveles permisibles de mercurio (35), en tanto que en Zaragoza fue de 7,1\%, en Segovia de $10,2 \%$ y en Remedios de 5,6 \% (cuadro 2).

\section{Mercurio total en la orina}

La concentración promedio de mercurio total en la orina fue de $3,88 \mu \mathrm{g} / \mathrm{dl}( \pm 4,41)$ y el $6,7 \%$ de las muestras superó el límite tolerado (37), el cual fue mayor en las mujeres lactantes residentes en el municipio de Segovia $(4,56 \mu \mathrm{g} / \mathrm{dl} \pm 4,88)$, seguido de El Bagre $(4,1 \mu \mathrm{g} / \mathrm{dl} \pm 4,7)$, de Zaragoza $(2,82 \mu \mathrm{g} /$ $\mathrm{dl} \pm 3,92)$, y, por último, de Remedios $(3,04 \mu \mathrm{g} / \mathrm{dl}$ $\pm 2,05$ ). El porcentaje de muestras de orina que superaron los límites permisibles de mercurio varió entre $12,2 \%$, en el municipio de Segovia, y 3,6\% en Zaragoza; sin embargo, en Remedios ninguna de las muestras superó el límite permisible (37) (cuadro 2).

\section{Mercurio total en el cabello}

Con respecto a la concentración de mercurio en el cabello, se determinó un promedio de 1,25 $\mu \mathrm{g} /$ $\mathrm{kg}( \pm 1,61)$, y el $35,3 \%$ de las muestras de cabello superó el límite permisible (36). En el municipio de El Bagre, la concentración de mercurio en el cabello registró un promedio de $1,26 \mu \mathrm{g} / \mathrm{kg}( \pm 1,86)$, y $32,7 \%$ las muestras superó el límite permisible, en tanto que, en Zaragoza, el promedio fue de 1,10 $\mu \mathrm{g} / \mathrm{kg}( \pm 1,48)$, y $35,7 \%$ de las muestras superó el límite permisible (36). Además, en Zaragoza se evidenció una correlación positiva y significativa entre las concentraciones de mercurio en el cabello y la de mercurio total en la leche materna, pero no así con las concentraciones de mercurio en la orina (cuadro 2).

En el municipio de Segovia, la concentración promedio de mercurio en el cabello fue mayor que en los demás municipios $(1,58 \mu \mathrm{g} / \mathrm{kg}, \pm 1,65)$, y $49 \%$ de las muestras superó el límite permisible. Asimismo, se evidenció una correlación positiva y significativa entre las diferentes matrices evaluadas (orina y cabello) con la concentración de mercurio total en la leche materna (cuadro 2).

Las mujeres residentes en el municipio de Remedios presentaron una concentración promedio de mercurio en cabello de $0,65 \mu \mathrm{g} / \mathrm{kg}( \pm 0,29)$, asimismo, el $11 \%$ de las muestras de cabello superó los límites permisibles. A diferencia de lo observado en los demás municipios, no se evidenció una correlación entre las diferentes matrices evaluadas (orina y cabello) y la concentración de mercurio total en la leche materna (cuadro 2).

Sin embargo, en general se evidenció una correlación positiva y significativa entre las diferentes

Cuadro 2. Concentraciones de mercurio en las diferentes matrices evaluadas en mujeres lactantes de dos municipios auríferos de Antioquia

\begin{tabular}{|c|c|c|c|c|c|c|}
\hline & & $\begin{array}{c}\text { Media } \\
\text { (desviación } \\
\text { estándar) }\end{array}$ & $\begin{array}{c}\text { Mediana } \\
\text { (rango } \\
\text { intercuartílico) }\end{array}$ & $\%(n)^{*}$ & $\begin{array}{l}\text { Correlación } \\
\text { con leche } \\
\text { materna }^{\star \star}\end{array}$ & $\mathbf{p}$ \\
\hline \multirow[t]{3}{*}{ General } & Concentración de mercurio en el cabello & $1,25 \quad(1,61)$ & $0,83(0,7)$ & $53 \quad(35,3)$ & $0,280^{\star *}$ & 0,001 \\
\hline & Concentración de mercurio en la orina & $3,88 \quad(4,41)$ & $2,69(3,18)$ & $6,7(10)$ & $0,618^{\star *}$ & 0,000 \\
\hline & Concentración de mercurio en la leche madre & $2,11 \quad(7,17)$ & $0,8 \quad(0,7)$ & $11,3(17)$ & 1 & \\
\hline \multirow[t]{3}{*}{ El Bagre } & Concentración de mercurio en el cabello & $1,26 \quad(1,86)$ & $0,68(0,8)$ & $32,7(18)$ & 0,273 & 0,044 \\
\hline & Concentración de mercurio en la orina & $4,1 \quad(4,7)$ & $2,61(2,93)$ & $5,5 \quad(3)$ & $0,789^{\star *}$ & 0,000 \\
\hline & Concentración de mercurio en la leche madre & $3,42(11,34)$ & $0,64(0,9)$ & $16,4 \quad(9)$ & 1 & \\
\hline \multirow[t]{3}{*}{ Zaragoza } & Concentración de mercurio en el cabello & $1,10(1,48)$ & $0,67(0,6)$ & $32,1 \quad(9)$ & 0,436 & 0,020 \\
\hline & Concentración de mercurio en la orina & $2,82 \quad(3,92)$ & $1,44(2,55)$ & $3,6 \quad(1)$ & 0,328 & 0,095 \\
\hline & Concentración de mercurio en la leche madre & $0,8 \quad(0,63)$ & $0,75(0,8)$ & $7,1 \quad(2)$ & 1 & \\
\hline \multirow[t]{3}{*}{ Segovia } & Concentración de mercurio en el cabello & $1,58 \quad(1,65)$ & $0,99(0,6)$ & $49 \quad(24)$ & $0,594^{\star *}$ & 0,000 \\
\hline & Concentración de mercurio en la orina & $4,56 \quad(4,88)$ & $3,33(4,39)$ & $12,2 \quad(6)$ & $0,724^{\star \star}$ & 0,000 \\
\hline & Concentración de mercurio en la leche madre & $1,76 \quad(3,35)$ & $0,9(0,6)$ & $10,2 \quad(5)$ & 1 & \\
\hline \multirow[t]{3}{*}{ Remedios } & Concentración de mercurio en el cabello & $0,65 \quad(0,29)$ & $0,57(0,4)$ & $11 \quad(2)$ & 0,035 & 0,890 \\
\hline & Concentración de mercurio en la orina & $3,04 \quad(2,05)$ & $2,83(3,62)$ & 0 & 0,032 & 0,903 \\
\hline & Concentración de mercurio en la leche madre & $1,12(0,71)$ & $0,99(0,4)$ & $5,6 \quad(1)$ & 1 & \\
\hline
\end{tabular}

* Porcentaje de mujeres que superó el límite permisible de la concentración de mercurio en cada una de las matrices evaluadas

${ }^{* *}$ Correlaciones de Spearman; la distribución no fue normal para ninguna de las concentraciones. 
matrices evaluadas (orina y cabello) y la concentración de mercurio total en la leche materna (cuadro 2).

\section{Discusión}

Esta investigación es la primera en que se evalúan las concentraciones de mercurio en la leche materna en poblaciones mineras de Colombia, específicamente en las zonas de explotación de oro de Antioquia. Es importante resaltar que la revisión sistemática de la literatura científica permitió detectar únicamente tres estudios $(23,38,39)$ que evaluaron las concentraciones de mercurio en leche materna en zonas mineras: uno de ellos en Brasil (38), otro en áreas mineras de Indonesia, Tanzania y Zimbabue (23), y el tercero en Filipinas (39).

Los resultados de este estudio sugieren el siguiente perfil de las características de las mujeres lactantes con altas concentraciones de mercurio en la leche materna: mujeres solteras o separadas, con estudios primarios, primíparas y sin antecedentes de aborto, que han tenido malaria, y llevan amalgamas en los dientes, y que, además, habitan viviendas con condiciones precarias y no tienen servicios públicos domiciliarios, viven a menos de $50 \mathrm{~m}$ de un sitio de compra o un beneficiadero, y en algunas de sus viviendas se quema oro y se almacena mercurio.

Los hallazgos del presente estudio ponen en evidencia la transferencia de mercurio inorgánico $(\mathrm{Hg}$ metálico) y orgánico (metilmercurio) del ambiente (entendido este como el microambiente y el mesoambiente) a los alimentos que consumen las madres y, en consecuencia, a la leche materna. La transferencia de mercurio inorgánico ocurre cuando la madre se expone a altas concentraciones de vapores de mercurio metálico debido a la cercanía de la vivienda a lugares de compra de oro, así como a la quema de amalgama de mercurio dentro de la vivienda. Por otra parte, la transferencia de mercurio orgánico se asocia con el consumo de alimentos, en especial el pescado, el cual constituye la principal fuente de proteína en la dieta materna, así como con la poca variedad en la alimentación, especialmente la escasa ingestión de otros alimentos cárnicos y de frutas.

En el presente estudio, el promedio $(2,11 \mu \mathrm{g} / \mathrm{L}, \pm$ desviación estándar, 5,17$)$ y la mediana $(0,8 \mu \mathrm{g} / \mathrm{L})$ de la concentración de mercurio total en la leche materna estuvieron por debajo de lo reportado en estudios anteriores realizados en Brasil, Indonesia, Tanzania, Zimbabue y Filipinas; sin embargo, la diferencia en las escalas usadas para medir la concentración de mercurio puede dificultar la comparación de estos hallazgos.

En el caso de la investigación en Brasil, realizada con un grupo de 47 mujeres lactantes que vivían en diferentes poblaciones y casas alrededor de río Madeira en la Amazonia (38), se observó un promedio de mercurio en la leche materna de 5,8 $\mathrm{ng} / \mathrm{g}(+5,9)$, en tanto que en Indonesia, Tanzania y Zimbabue, se evaluaron 46 mujeres lactantes que vivían en las áreas mineras de Kalimantan y Sulawesi (Indonesia), Geita (Tanzania) y Kadoma (Zimbabue) seleccionadas de manera aleatoria, y se encontró que $40 \%$ de ellas superaba los límites permisibles de mercurio en leche materna (23) establecidos por la Agency for Toxic Substances and Disease Registry (ATSDR) del Departamento de Salud de los Estados Unidos (35), porcentaje superior al observado en el presente estudio $(11,3 \%)$, con una mediana igualmente superior $(1,87 \mu \mathrm{g} / \mathrm{L})$.

En el estudio de Filipinas se evaluaron 78 madres habitantes de la zona minera de Tagum y sus hijos, cuya concentración promedio de mercurio fue de $36 \mathrm{ppb}$, cifra que no es comparable con la del presente estudio, pues las mediciones se hicieron con escalas diferentes (39).

La mayoría de los estudios que evalúan las concentraciones de mercurio en la leche materna, se han hecho en poblaciones pesqueras, no en comunidades mineras; en estas se ha observado una gran variabilidad de las concentraciones, pero, en general, han sido inferiores $(21,40-45)$ a las concentraciones encontradas en el presente estudio. Solo en el caso de Al-Saleh, et al., en Arabia Saudita (20), y en zonas pesqueras de áreas con uso intensivo de mercurio en Brasil (12), se han registrado cifras mayores.

En el presente estudio se observó que las concentraciones de mercurio total en la orina de las madres lactantes superaron las concentraciones reportadas por Al-Saleh, et al. $(46,47)$, en Arabia Saudita (2013 y 2016) y Vimy, et al. (48), en Canadá, diferencias que pueden deberse a que la población de estos reportes estaba constituida por mujeres lactantes que residían en zonas pesqueras y no en zonas mineras.

Por el contrario, los hallazgos de Gaxiola-Robles, et al. (49), en México y de Bose-O'Reilly, et al. (23). en Indonesia, Tanzania y Zimbabue son superiores a las concentraciones halladas en las mujeres 
lactantes del presente estudio, lo cual se explicaría porque, aunque estos estudios se llevaron a cabo en zonas mineras, las madres lactantes estaban expuestas de forma directa a los vapores de mercurio, ya que trabajaban en labores mineras y de quema de amalgama, mientras que en el presente estudio ninguna mujer lactante trabajaba en tales labores en el momento de la encuesta.

La excreción de mercurio en la leche materna pone en riesgo la salud del niño lactante, pues el sistema nervioso en desarrollo es más vulnerable a los efectos del metilmercurio, incluso cuando se trata de dosis bajas $(5,50)$. Después de una exposición prenatal y posnatal temprana al metilmercurio se han detectado efectos en procesos tan críticos como la división celular (induce la detención mitótica) y la migración celular (alteración del patrón de movimiento de las células) (51). Los efectos del metilmercurio en el sistema nervioso en desarrollo pueden variar desde retrasos sutiles de desarrollo cognitivo y motor hasta la parálisis cerebral, según la dosis y el tiempo de exposición de la madre durante el embarazo (5).

La exposición leve a moderada al metilmercurio durante la gestación y la lactancia puede resultar en síntomas tardíos que no se observan en el momento del nacimiento, tales como dificultad para caminar y hablar, y la persistencia de los reflejos perinatales anormales $(19,31)$, aunque en algunos estudios no se han encontrado efectos en el desarrollo neurológico cuando la exposición ha sido baja $(18,52,53)$, lo cual evidencia la gran variabilidad de los efectos (54). Por otro parte, la exposición elevada genera alteraciones como microcefalia, hiperreactividad bronquial, deficiencias en la motricidad gruesa, deficiencias mentales, ceguera, sordera, parálisis cerebral con retraso mental, dificultades en la alimentación y déficit motor importante (55).

En cuanto a la relación entre la toxicidad del mercurio metálico y el desarrollo neurológico, las evidencias son escasas; se ha evidenciado que en niños que viven en áreas de explotación minera de oro y uso extensivo de mercurio aparecen alteraciones en el neurodesarrollo y en la respuesta evocada a estímulos auditivos (56).

Con respecto a las concentraciones de mercurio total en el cabello de la madre, estas fueron más altas que las evidenciadas en los estudios de Gundacker, et al. (57), en Austria, y de Miklavčič, et al. $(42,58)$, en Italia, Eslovenia y Croacia, pero más bajas que las observadas por Barbosa, et al.
(38), y Vieira, et al. (12), en Brasil, Miklavčič, et al. (58), en Grecia y Bose-O'Reilly, et al. (23), en Indonesia, Tanzania y Zimbabue. Estos hallazgos se relacionan con el grado de contaminación con metilmercurio en peces y ríos, lo cual sugiere que, en las zonas mineras de estos países, se registra una gran contaminación generada por la producción de lixiviados con altas concentraciones de mercurio, la precipitación de vapores de mercurio con las lluvias, los procesos de escorrentía y de acumulación en los sedimentos de ríos y lagunas, donde el material orgánico permite su transformación y acumulación tanto en los peces como en las plantas regadas o rociadas con estas aguas o por ellas.

En cuanto a las correlaciones observadas en este estudio entre la concentración de mercurio en la leche materna y la registrada en el cabello y la orina, estas concuerdan con lo encontrado en estudios en Arabia Saudita (47), Indonesia, Italia (43), Tanzania, Zimbabue (23), Filipinas, Japón (59) y Canadá (48), pero no así con las registradas en China (44), en otro estudio en Japón (49) y en la zona minera de Brasil (38), en los cuales no se apreció ninguna correlación entre las diferentes matrices evaluadas y la concentración de mercurio en la leche materna.

Los hallazgos del presente estudio ponen en evidencia una correlación positiva y significativa entre las diferentes matrices evaluadas (orina y cabello) y la concentración de mercurio total en leche materna. El promedio de mercurio en la leche materna, la orina y el cabello fueron de $2,11 \mu \mathrm{g} / \mathrm{dl}$ $( \pm 7,17), 3,88 \mu \mathrm{g} / \mathrm{dl}( \pm 4,41)$, y $1,25 \mu \mathrm{g} / \mathrm{kg}( \pm 1,61)$, respectivamente, lo cual permitiría concluir que se registró la transferencia de mercurio inorgánico (Hg metálico) y orgánico (metilmercurio) a la leche materna desde el ambiente y mediante los alimentos consumidos por las madres.

\section{Conflicto de intereses}

Los autores manifestamos que no tenemos ningún conflicto de intereses con respecto al contenido del manuscrito.

\section{Financiación}

Esta publicación es resultado de la investigación realizada con el apoyo financiero de la estrategia de sostenibilidad 2013-2014 del Comité para el Desarrollo de la Investigación, CODI, para los grupos de Seguridad y Salud en el Trabajo y Epidemiología, adscritos a la Facultad Nacional de Salud Pública de la Universidad de Antioquia. 


\section{Referencias}

1. Crespo-López ME, Herculano AM, Corvelo TC, Do Nascimento JL. Mercurio y neurotoxicidad. Rev Neurol. 2005;40:441-7.

2. Clarkson TW, Magos L. The toxicology of mercury and its chemical compounds. Crit Rev Toxicol. 2006;36:609-62. https://doi.org/10.1080/10408440600845619

3. Clarkson TW, Magos L, Myers GJ. The toxicology of mercury --current exposures and clinical manifestations. N Engl J Med. 2003;349:1731-7. https://doi.org/10.1056/ NEJMra022471

4. Clarkson TW. The three modern faces of mercury. Environ Health Perspect. 2002;110(Suppl.1):11-23.

5. Castoldi AF, Johansson C, Onishchenko N, Coccini T, Roda E, Vahter M, et al. Human developmental neurotoxicity of methylmercury: Impact of variables and risk modifiers. Regul Toxicol Pharmacol. 2008;51:201-14. https://doi.org/10.1016/j.yrtph.2008.01.016

6. Oskarsson A, Palminger Hallén I, Sundberg J, Petersson Grawé K. Risk assessment in relation to neonatal metal exposure. Analyst. 1998;123:19-23.

7. Castilhos ZC, Rodrigues-Filho S, Rodrigues APC, VillasBôas RC, Siegel S, Veiga MM, et al. Mercury contamination in fish from gold mining areas in Indonesia and human health risk assessment. Sci Total Environ. 2006;368:320-5. https://doi.org/10.1016/j.scitotenv.2006.01.039

8. Li P, Feng XB, Qiu GL, Shang LH, Li ZG. Mercury pollution in Asia: A review of the contaminated sites. $J$ Hazard Mater. 2009;168:591-601. https://doi.org/10.1016/j. jhazmat.2009.03.031

9. Cortés-Maramba N, Reyes JP, Francisco-Rivera AT, Akagi H, Sunio R, Panganiban LC. Health and environmental assessment of mercury exposure in a gold mining community in Western Mindanao, Philippines. J Environ Manage. 2006;81:126-34. https://doi.org/10.1016/j.jenvman. 2006.01.019

10. Drasch G, Böse-O'Reilly S, Beinhoff C, Roider G, Maydl S. The Mt. Diwata study on the Philippines 1999 --assessing mercury intoxication of the population by small scale gold mining. Sci Total Environ. 2001;267:151-68. https://doi. org/10.1016/S0048-9697(00)00806-8

11. Steckling N, Boese-O'Reilly S, Gradel C, Gutschmidt K, Shinee E, Altangerel E, et al. Mercury exposure in female artisanal small-scale gold miners (ASGM) in Mongolia: An analysis of human biomonitoring (HBM) data from 2008. Sci Total Environ. 2011;409:994-1000. https://doi.org/10.1016/j. scitotenv.2010.11.029

12. Vieira SM, de Almeida R, Holanda IB, Mussy MH, Galvão RC, Crispim PT, et al. Total and methyl-mercury in hair and milk of mothers living in the city of Porto Velho and in villages along the Rio Madeira, Amazon, Brazil. Int J Hyg Environ Health. 2013;216:682-9. https://doi.org/10.1016/j. ijheh.2012.12.011

13. Li P, Feng X, Shang L, Qiu G, Meng B, Zhang H, et al. Human co-exposure to mercury vapor and methylmercury in artisanal mercury mining areas, Guizhou, China. Ecotoxicol Environ Saf. 2011;74:473-9. https://doi.org/10.1016/j. ecoenv.2010.10.030
14. Cordy P, Veiga MM, Salih I, Al-Saadi S, Console S, García $\mathbf{O}$, et al. Mercury contamination from artisanal gold mining in Antioquia, Colombia: The world's highest per capita mercury pollution. Sci Total Environ. 2011;410:15460. https://doi.org/10.1016/j.scitotenv.2011.09.006

15. Cordy P, Veiga M, Crawford B, García O, González V, Moraga D, et al. Characterization, mapping, and mitigation of mercury vapour emissions from artisanal mining gold shops. Environ Res. 2013;125:82-91. https://doi. org/10.1016/j.envres.2012.10.015

16. Ministerio de Minas y Energía. Anuario estadístico minero de Colombia. Fecha de consulta: 28 de noviembre de 2016. Disponible en: https://www.minminas.gov.co/anuarioestadistico-minero

17. América Economía. El lado oscuro y venenoso del boom del oro en Colombia. Fecha de consulta: 28 de noviembre de 2016. Disponible en: http://www.americaeconomia.com/ negocios-industrias/el-lado-oscuro-y-venenoso-del-boomdel-oro-en-colombia

18. Jensen TK, Grandjean P, Jørgensen EB, White RF, Debes F, Weihe P. Effects of breast feeding on neuropsychological development in a community with methylmercury exposure from seafood. J Expo Anal Environ Epidemiol. 2005;15: 423-30. https://doi.org/10.1038/sj.jea.7500420

19. Marques RC, Dórea JG, Bernardi JVE, Bastos WR, Malm O. Prenatal and postnatal mercury exposure, breastfeeding and neurodevelopment during the first 5 years. Cogn Behav Neurol. 2009;22:134-41. https://doi.org/10.1097/ WNN.0b013e3181a72248

20. Al-Saleh I, Shinwari N, Mashhour A. Heavy metal concentrations in the breast milk of Saudi women. Biol Trace Elem Res. 2003;96:21-37. https://doi.org/10.1385/ BTER:96:1-3:21

21. García-Esquinas E, Pérez-Gómez B, Fernández MA, Pérez-Meixeira AM, Gil E, de Paz C, et al. Mercury, lead and cadmium in human milk in relation to diet, lifestyle habits and sociodemographic variables in Madrid (Spain). Chemosphere. 2011;85:268-76. https://doi.org/10.1016/j. chemosphere.2011.05.029

22. Yang J, Jiang Z, Wang Y, Qureshi IA, Wu XD. Maternalfetal transfer of metallic mercury via the placenta and milk. Ann Clin Lab Sci. 1997;27:135-41.

23. Bose-O'Reilly S, Lettmeier B, Roider G, Siebert U, Drasch G. Mercury in breast milk - a health hazard for infants in gold mining areas? Int J Hyg Environ Health. 2008;211:615-23. https://doi.org/10.1016/j.ijheh.2007.09.015

24. Veiga MM, Baker RF. Protocols for environmental and health assessment of mercury released by artisanal and small-scale gold miners. Vienna, Austria: GEF/UNDP/ UNIDO; 2004. p. 77-155.

25. United Nations Environment Programme (UNEP). Guidance for identifying populations at risk from mercury exposure. Geneva: UNEP; 2010. p. 119-50.

26. Oskarsson A, Schültz A, Skerfving S, Hallén IP, Ohlin B, Lagerkvist BJ. Total and inorganic mercury in breast milk in relation to fish consumption and amalgam in lactating women. Arch Environ Health. 1996;51:234-41._https://doi.or g/10.1080/00039896.1996.9936021 
27. da Costa SL, Malm O, Dórea JG. Breast-milk mercury concentrations and amalgam surface in mothers from Brasilia, Brazil. Biol Trace Elem Res. 2005;106:145-51. https://doi.org/10.1385/BTER:106:2:145

28. Vahter M, Akesson A, Lind B, Björs U, Schütz A, Berglund M. Longitudinal study of methylmercury and inorganic mercury in blood and urine of pregnant and lactating women, as well as in umbilical cord blood. Environ Res. 2000;84:186-94. https://doi.org/10.1006/enrs.2000.4098

29. Okati N, Sari AE, Ghasempouri SM. Hair mercury concentrations of lactating mothers and breastfed infants in Iran (fish consumption and mercury exposure). Biol Trace Elem Res. 2012;149:155-62. https://doi.org/10.1007/ s12011-012-9424-7

30. Norouzi E, Bahramifar N, Ghasempouri SM. Effect of teeth amalgam on mercury levels in the colostrums human milk in Lenjan. Environ Monit Assess. 2012;184:375-80. https://doi.org/10.1007/s10661-011-1974-1

31. Chien L-C, Han B-C, Hsu C-S, Jiang C-B, You H-J, Shieh M-J, et al. Analysis of the health risk of exposure to breast milk mercury in infants in Taiwan. Chemosphere. 2006;64:7985. https://doi.org/10.1016/j.chemosphere.2005.11.059

32. Behrooz RD, Esmaili-Sari A, Peer FE, Amini M. Mercury concentration in the breast milk of Iranian women. Biol Trace Elem Res. 2012;147:36-43. https://doi.org/10.1007/ s12011-011-9283-7

33. McDowell MA, Dillon CF, Osterloh J, Bolger PM, Pellizzari E, Fernando R, et al. Hair mercury levels in U.S. children and women of childbearing age: Reference range data from NHANES 1999-2000. Environ Health Perspect. 112:1165-71. https://doi.org/10.1289/ehp.7046

34. United States Environmental Protection Agency EPA. Method 7473 (SW-846): Mercury in solids and solutions by thermal decomposition, amalgamation, and atomic absorption spectrophotometry | Homeland Security Research | US EPA. Fecha de consulta: 30 de noviembre de 2016. Disponible en: https://www.epa.gov/homelandsecurity-research/epa-method-7473-sw-846-mercurysolids-and-solutions-thermal-decomposition

35. Agency for Toxic Substances and Disease Registry. ATSDR Tox Profiles 2004. Atlanta, GA: US Department of Health and Human Services; 2004. p. 445.

36. Committee on the Toxicological Effects of Methylmercury. Toxicological effects of methylmercury. National Academy of Sciences, 2000. Fecha de consulta: 28 de noviembre de 2016. Disponible en: https://www.ncbi.nlm.nih.gov/books/ NBK225773/

37. American Conference of Governmental Industrial Hygienists (ACGIH $\AA$ ). TLV ${ }^{\circledR}$ and $B E I \circledast$ guidelines. Cincinnati: ACGIH; 2015. p. 9-97.

38. Barbosa AC, Silva SR, Dórea JG. Concentration of mercury in hair of indigenous mothers and infants from the Amazon basin. Arch Environ Contam Toxicol.1998;34:100-5.

39. Ramírez GB, Cruz MC, Pagulayan O, Ostrea E, Dalisay C. The Tagum study I: Analysis and clinical correlates of mercury in maternal and cord blood, breast milk, meconium, and infants' hair. Pediatrics. 2000;106:774-81. https://doi. org/10.1542/peds.106.4.774
40. Sharma R, Pervez S. Toxic metals status in human blood and breast milk samples in an integrated steel plant environment in Central India. Environ Geochem Health. 2005;27:39-45. https://doi.org/10.1007/s10653-004-1628-0

41. Björnberg KA, Vahter $M$, Berglund B, Niklasson B, Blennow M, Sandborgh-Englund G. Transport of methylmercury and inorganic mercury to the fetus and breast-fed infant. Environ Health Perspect. 2005;113:13815._https://doi.org/10.1289/ehp.7856

42. Miklavčič A, Cuderman P, Mazej D, Snoj Tratnik J, Krsnik $\mathbf{M}$, Planinšek $\mathbf{P}$, et al. Biomarkers of low-level mercury exposure through fish consumption in pregnant and lactating Slovenian women. Environ Res. 2011;111:1201-7._https:// doi.org/10.1016/j.envres.2011.07.006

43. Valent F, Mariuz M, Bin M, Little D, Mazej D, Tognin V, et al. Associations of prenatal mercury exposure from maternal fish consumption and polyunsaturated fatty acids with child neurodevelopment: A prospective cohort study in Italy. J Epidemiol. 2013;23:360-70. https://doi.org/10.2188/ jea.JE20120168

44. Li M, Wu M, Xu J, Du J, Yan C. Body burden of hg in different bio-samples of mothers in Shenyang city, China. PLoS ONE. 2014;9:e98121._https://doi.org/10.1371/journal. pone.0098121

45. De Felip E, Bianchi F, Bove C, Cori L, D'Argenzio A, D’Orsi G, et al. Priority persistent contaminants in people dwelling in critical areas of Campania Region, Italy (SEBIOREC biomonitoring study). Sci Total Environ. 2014;487:420-35. https://doi.org/10.1016/j.scitotenv.2014.04.016

46. Al-Saleh I, Abduljabbar M, Al-Rouqi R, Elkhatib R, Alshabbaheen A, Shinwari N. Mercury $(\mathrm{Hg})$ exposure in breast-fed infants and their mothers and the evidence of oxidative stress. Biol Trace Elem Res. 2013;153:145-54. https://doi.org/10.1007/s12011-013-9687-7

47. Al-Saleh I, Nester M, Abduljabbar M, Al-Rouqi R, Eltabache C, Al-Rajudi T, et al. Mercury $(\mathrm{Hg})$ exposure and its effects on Saudi breastfed infant's neurodevelopment. Int J Hyg Environ Health. 2016;219:129-41. https://doi. org/10.1016/j.ijheh.2015.10.002

48. Vimy MJ, Hooper DE, King WW, Lorscheider FL. Mercury from maternal "silver» tooth fillings in sheep and human breast milk. A source of neonatal exposure. Biol Trace Elem Res.1997;56:143-52._https://doi.org/10.1007/BF02785388

49. Gaxiola-Robles R, Labrada-Martagón V, Celis de la Rosa A de J, Acosta-Vargas B, Méndez-Rodríguez LC, Zenteno-Savín T. Interaction between mercury $(\mathrm{Hg})$, arsenic (As) and selenium (Se) affects the activity of glutathione S-transferase in breast milk; possible relationship with fish and sellfish intake. Nutr Hosp. 2014;30:436-46. https://doi. org $/ 10.3305 /$ nh.2014.30.2.7441

50. Boischio AA, Henshel DS. Linear regression models of methyl mercury exposure during prenatal and early postnatal life among riverside people along the upper Madeira river, Amazon. Environ Res. 2000;83:150-61. https://doi. org/10.1006/enrs.2000.4050

51. Clarkson TW. Metal toxicity in the central nervous system. Environ Health Perspect. 1987;75:59-64. https://doi.org/10. $2307 / 3430577$ 
52. Daniels JL, Longnecker MP, Rowland AS, Golding J, ALSPAC Study Team. University of Bristol Institute of Child Health. Fish intake during pregnancy and early cognitive development of offspring. Epidemiology. 2004;15:394402. https://doi.org/10.1097/01.ogx.0000151643.24743.40

53. Tatsuta N, Nakai K, Murata K, Suzuki K, Iwai-Shimada $\mathbf{M}$, Yaginuma-Sakurai $\mathrm{K}$, et al. Prenatal exposures to environmental chemicals and birth order as risk factors for child behavior problems. Environ Res. 2012;114:47-52. https://doi.org/10.1016/j.envres.2012.02.001

54. Pugach S, Clarkson T. Prenatal mercury exposure and postnatal outcome: Clinical case report and analysis. Clin Toxicol (Phila). 2009;47:366-70. https://doi.org/10.1080/ 15563650902866911

55. Ibrahim D, Froberg B, Wolf A, Rusyniak DE. Heavy metal poisoning: Clinical presentations and pathophysiology. Clin Lab Med. 2006;26:67-97. https://doi.org/10.1016/j.cll. 2006.02.003
56. Counter SA. Neurophysiological anomalies in brainstem responses of mercury-exposed children of Andean gold miners. J Occup Environ Med. 2003; 45:87-95. https://doi. org/10.1097/00043764-200301000-00017

57. Gundacker C, Fröhlich S, Graf-Rohrmeister K, Eibenberger B, Jessenig V, Gicic D, et al. Perinatal lead and mercury exposure in Austria. Sci Total Environ. 2010;408:5744-9. https://doi.org/10.1016/j.scitotenv.2010.07.079

58. Miklavčič A, Casetta A, Snoj Tratnik J, Mazej D, Krsnik M, Mariuz M, et al. Mercury, arsenic and selenium exposure levels in relation to fish consumption in the Mediterranean area. Environ Res. 2013;120:7-17. https://doi.org/10.1016/j. envres.2012.08.010

59. Iwai-Shimada M, Satoh H, Nakai K, Tatsuta N, Murata K, Akagi H. Methylmercury in the breast milk of Japanese mothers and lactational exposure of their infants. Chemosphere. 2015;126:67-72. https://doi.org/10.1016/j. chemosphere.2014.12.086 